\title{
Sensing proteins outside of the box
}

\author{
F. Gisou van der Goot and Stefan Matile
}

The stochastic sensing of single molecules passing though ion channels has now grown into a mature field ${ }^{1}$. Today's channel sensor of choice, $\alpha$-hemolysin $(\alpha \mathrm{HL})$, has been engineered extensively (and very successfully) to perceive molecules of increasing size and complexity. This new, general, and elegant analytical technique has, however, been limited to molecules that can pass through the $\alpha H L$ channel. Because this is not the case for central metabolites such as double-stranded DNA or native proteins, it seemed that either suitable pores larger than $\alpha H L$ would have to be constructed, a troublesome task, or macromolecules could only be sensed in a denatured form. In this issue, Bayley and coworkers ${ }^{2}$ have solved the fundamental problem of sensing macromolecules larger than the ion channel diameter ${ }^{2}$.

In an approach related to the design of gramicidin ion channel sensors on solid support ${ }^{3}$, they have designed a strategy for stochastic sensing of molecules that exist outside the $\alpha \mathrm{HL}$ channel that is simple and employs a more stable ion channel as a sensor. They covalently attached a simple polyethylene glycol (PEG) chain to one of the $\alpha \mathrm{HL}$ monomers within the heptameric pore-forming complex (Fig. 1), and tethered a ligand to the other end. Once the $\alpha \mathrm{HL}$ channel formed in a planar lipid bilayer, the tethered PEG chain was able to move through the channel from one side of the bilayer to the other, which led to electrical flickering of the channel. Subsequent binding of a protein to the ligand abolished this flickering by trapping the ligand on one side or the other of the membrane. When the binding protein was added to the same side as the extramembraneous part of the $\alpha \mathrm{HL}$ channel, the PEG chain adopted a stretched conformation oriented toward the outside of the pore like the leash of a dog running away from his master (Fig. 1A). In this case, pore conductance is unchanged. More intriguingly, when the binding protein is added to the opposite side of the membrane, binding to the ligand blocks the PEG polymer in a transmembrane conformation (Fig. 1B). In this case, pore conductance is reduced by partial obstruction of the pore.

This protein sensing strategy has been developed using biotin as the ligand, and

F. Gisou van der Goot and Stefan Matile are in the Departments of Biochemistry and Organic Chemistry, University of Geneva, 30, quai E. Ansermet, CH-1211 Geneva 4, Switzerland (gisou.vandergoot@biochem.unige.ch and stefan.matile@chiorg.unige.ch).

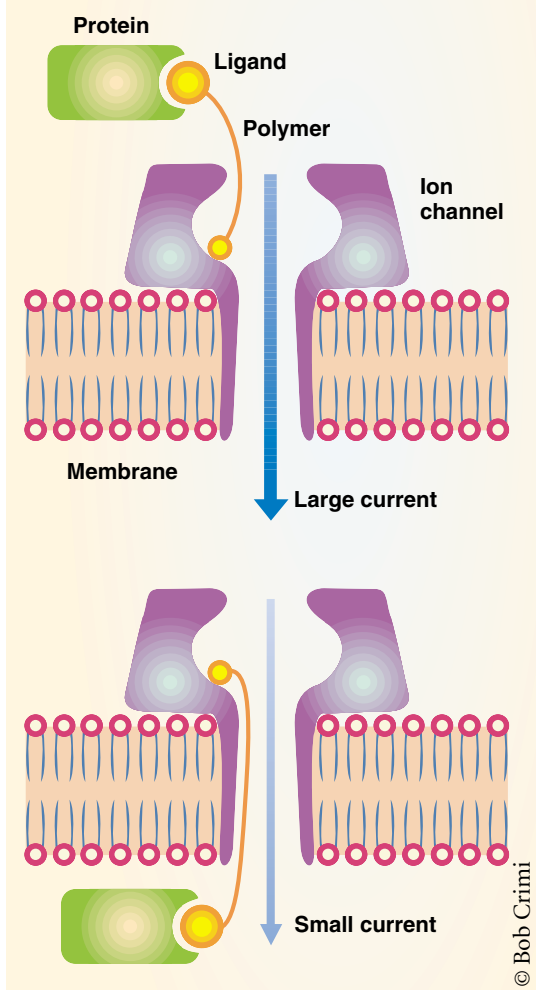

Figure 1. A protein channel for sensing external molecules. A membrane-spanning ion channel is equipped with a polymer chain that carries a ligand at the other end. (A) Binding of the ligand to a protein added to the same side of the membrane does not reduce the current flowing through the ion channel. (B) Binding of the ligand to a protein added to the other side of the membrane reduces the current flowing through the ion channel.

either streptavidin, a lower binding affinity streptavidin mutant, and an anti-biotin antibody as binding proteins. The key finding is that the fundamental advantages of stochastic sensing inside ion channels-sensitivity, fast kinetics at the single-molecule level-are maintained in external sensing. This result is a great breakthrough because it extends the stochastic single-molecule sensing technique beyond its own apparent limits, and moreover, the approach can be generally applied.

The first time a biochemical reaction could be observed at a single-molecule level using an ion channel as the sensing device was the early 1990s, by Alan Finckelstein and coworkers ${ }^{4}$. Their initial aim was to probe the structure of the diphtheria toxin ion channel by introducing a single cysteine residue at a site thought to be in the mouth of the channel. After allowing this mutant to form channels in a planar lipid bilayer, a sulfhydryl-reactive probe was added to one of the compartments to add a positive or negative charge, depending on the probe used, to the cysteine residue. Not surprisingly, it was found that such covalent modification altered the electric current flowing through a single open ion channel. More surprisingly, at least at that time, it was noted that rate constants for this reaction could be obtained from the lag time between the appearance of an open ion channel and its chemical transformation. And it was realized that these kinetic measurements are sensitive (nanomolar concentrations), fast (up to microsecond resolution), and without delays from mixing and diffusion. The evident and appealing implications for fast enzyme kinetics at the singlemolecule level were, however, never pursued.

This may be the right time to reconsider Finckelstein's observations. Bayley's protein sensor could well be the key for the transition from sensing single molecular recognition events to sensing single chemical transformations. As long as sensing was limited to the interior of ion channels, there were convincing arguments why stochastic single-enzyme kinetics would be untenable, because engineering large catalytic units into the ion-conducting $\beta$-barrel of $\alpha \mathrm{HL}$ still seems unrealistic despite progress with catalytic barrels in water ${ }^{5}$. Even if such a catalytic ion channel could be constructed, would it be more than an isolated (though admittedly intriguing) curiosity, without significance for real-world enzymology?

With Bayley's protein sensor, things have changed. There is no fundamental technical problem that would prevent replacement of the tethered ligand in Figure 1 with a substrate of free choice, and there is obviously no problem in replacing the binding protein in Figure 1 with any enzyme. Although significant progress is being made toward DNA sequencing within ion channels ${ }^{6}$, it is likely that the next breakthrough in this field will however concern stochastic single-enzyme kinetics, a development that biologists, biochemists, and chemists can await with anticipation.

1. Bayley, H. \& Martin, C.R. Chem. Rev. $\mathbf{1 0 0}$ 2575-2594 (2000).

2. Movileanu, L., Howorka, S., Braha, O. \& Bayley, H. Nat. Biotechnol. 18, 1091-1095 (2000).

3. Cornell, B.A. et al. Nature 387, 580-583 (1997).

4. Mindell, J.A. et al. Proc. Natl. Acad. Sci. USA 91, 5272-5276 (1994).

5. Altamirano, M.M., Blackburn, J.M., Aguayo, C. \& Fersht, A.R. Nature 403, 617-622 (2000).

6. Meller, A. et al. Proc. Natl. Acad. Sci. USA 97 1079-1084 (2000). 